\title{
Probing the surface chemistry of single nanowire sensor in operando mode
}

\author{
M. Kazemian-Abyanehc ${ }^{1}$, M. Amati ${ }^{1}$, L. Gregoratti ${ }^{1}$, M. Kiskinova ${ }^{1}$, K. Benkstein ${ }^{2}$, \\ S. Semancik ${ }^{2}$ and A. Kolmakov ${ }^{3}$ \\ ${ }^{1}$ ELETTRA Sincrotrone Trieste 34149 Trieste, Italy \\ ${ }^{2}$ National Institute of Standards and Technology, Gaithersburg, MD 20899, USA \\ ${ }^{3}$ Southern Illinois University at Carbondale, Carbondale, Illinois 62901, USA \\ Corresponding e-mail address: akolmakov@physics.siu.edu
}

\begin{abstract}
The individual single crystalline and polycrystalline single $\mathrm{SnO}_{2}$ nanowire sensors have been fabricated on the base of the NIST micro-hot plates. Using Scanning Photoelectron Microscope (SPEM) with the lateral resolution of ca $80 \mathrm{~nm}$ we were able to access spectroscopicaly (XPS) the changes of surface chemistry of the individual nanowires during their operation. It was possible in real time to monitor the changes in conductance of the nanowire upon redox gas exposure and link them to the formation of the specific surface groups. The effect of crystallinity of the nanowires and electroactive defects on their sensing performance has been studied.
\end{abstract}

Key words: $\mathrm{SnO}_{2}$ nanowires, surface redox reaction, in situ microscopy, XPS in operando mode

\section{Introduction}

The surface reactivity and electron transport in metal oxide nanowire sensors have a strong dependence on their effective diameters and type of facets exposed to the ambient; therefore, the traditional analytical methods of probing the surface chemistry of the nanowire films with a broad size/shape distribution of nanowires will inevitably lead to the convoluted spectra reminiscent to common powder results. Therefore, to access the fundamentals of the surface chemistry and its coupling of with transport properties in these nanostructures, the experiments on individual nanostructure with clean and wellcharacterized facets of are required [1, 2]. Synchrotron Radiation- (SR-) based scanning photoelectron microscopy (SPEM), achieved the spatial resolution of $100 \mathrm{~nm}$ [3]. In this research, we combined this setup with state of the art micro-hot plate nanowire sensing platform[4]. This allows us to monitor the surface processes of the working nanosensor spectroscopically in operando regime.

\section{Experimental setup}

Two different types of the nanowires have been tested. Single crystal well faceted $\mathrm{SnO}_{2}$ nanowires and nanobelts were grown at ca $900{ }^{\circ} \mathrm{C}$ using standard VLS technique. For comparison polycrystalline $\mathrm{SnO}_{2}$ nanowires were obtained via controlled oxidation of $\mathrm{Sn}$ nanowires electro-deposited in to nanopores of porous alumina oxide. Figure 1a depicts the principles of the experimental and data acquisition setup. The detailed information for the NW surface chemical state and its lateral variations can be obtained from the photoelectron spectra and images of individual $\mathrm{SnO}_{2} \mathrm{NWs}$, measured by SPEM, where the incident photon beam is focused to a small spot (ca 100nm) (Fig.1a). SPEM can operate in two modes: (i) imaging by collecting photoelectrons within a selected kinetic energy window (Fig.1 b), while scanning the sample with respect to the focused beam and (ii) conventional energy dispersive electron spectroscopy from the illuminated local spot (micro-XPS) (Fig.2).

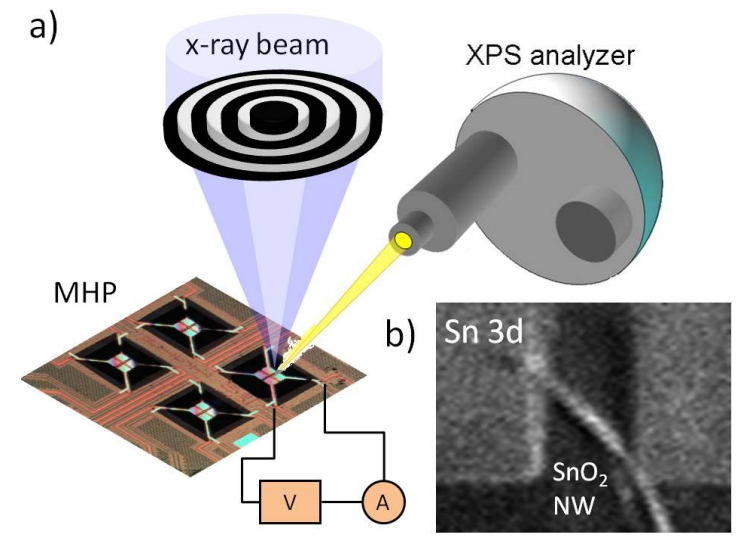

Fig.1 a) NIST micro-hot plates with nanowires bridging the electrodes. The nanowire sensing platform was coupled with zone plate based SPEM setup; b) photoelectron image of the individual nanowire sensor taken with $\mathrm{Sn} 3 d$ electrons. 


\section{Results}

Fig. $1 \mathrm{~b}$ displays XPS image of the individual nanowire in Sn 3d electrons. The homogeneous oxidation state of the most part of tin nanowire follows from the negligible variation of the gray scale along the length of the nanowire. Somewhat brighter central part of the nanowire is presumably due to local reduction of the nanowire by x-ray beam. Fig. 2 shows a representative set of $01 \mathrm{~s}$, (top spectra) and Sn 3d spectra (bottom panel) acquired from the individual $\mathrm{SnO}_{2}$ nanowire during exposure to molecular oxygen at ca 200 ${ }^{\circ} \mathrm{C}$.
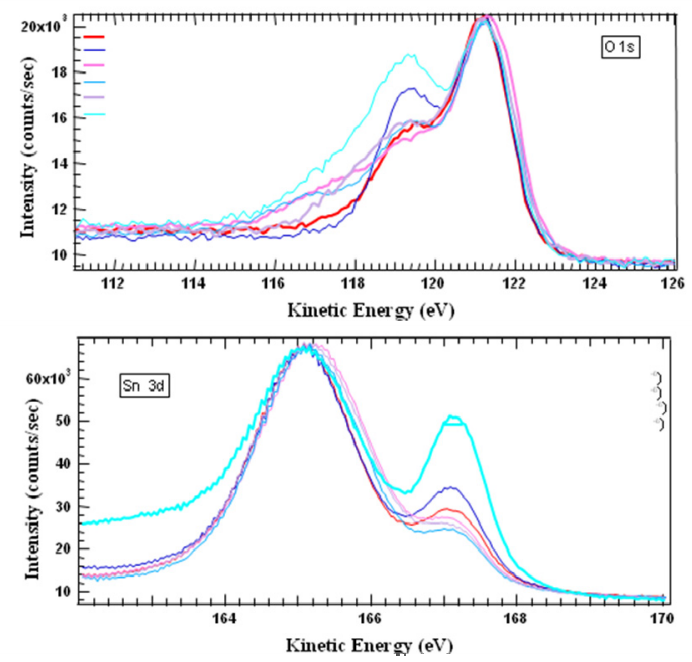

Fig. 2 Evolution of O 1s (top) and Sn 3d XPS spectra during the exposure of nanowire to molecular oxygen

As can be seen the relative ratio of $\mathrm{O}^{2+}$ and $\mathrm{O}^{4+}$ bands gradually changes upon dissociation of molecular oxygen and oxidation of the reduced oxide. Sn 3d spectral evolution follows the same trend indication the gradual reduction of $\mathrm{Sn}^{0}$ upon oxidation.

The observed evolutions of the chemical status of the nanowire surface correlates with simultaneous conductometric measurements. As an example, the gradual growth of chemisorbed oxygen (high binding energy shoulder in the Fig. 2 top panel) is concomitant with the conductance drop (not shown here).

\section{Summary}

The combination of SPEM spectromicroscopy on individual nanostructure with simultaneous conductometric studies allows one to have an access to fundamental surface processes of reducible oxides upon ionosorption/ dissociation of simple reducing $\left(\mathrm{H}_{2}, \mathrm{CO}\right)$ and oxidizing $\left(\mathrm{O}_{2}, \mathrm{NOx}\right)$ molecules, and model redox reactions ( $\mathrm{CO}$ and $\mathrm{H}_{2}$ oxidation). The evolution of electronic structure and concomitant adsorptivity of the nanostructures as a function of their crystallinity has been addressed as well.

\section{References}

[1] A. Kolmakov, S. Potluri, A. Barinov, T. O. Mentes, L. Gregoratti, M. A. Nino, A. Locatelli, and M. Kiskinova, "Spectromicroscopy for Addressing the Surface and Electron Transport Properties of Individual 1-D Nanostructures and Their Networks," Acs Nano, vol. 2, pp. 19932000, 2008.

[2] K. Katsiev, A. Kolmakov, M. H. Fang, and U. Diebold, "Characterization of individual $\mathrm{SnO} 2$ nanobelts with STM," Surface Science, vol. 602, pp. L112-L114, 2008.

[3] S. Gunther, B. Kaulich, L. Gregoratti, and M. Kiskinova, "Photoelectron microscopy and applications in surface and materials science," in Progress in Surface Science, vol. 70, 2002, pp. 187-260.

[4] D. C. Meier, S. Semancik, B. Button, E. Strelcov, and A. Kolmakov, "Coupling nanowire chemiresistors with MEMS microhotplate gas sensing platforms," Applied Physics Letters, vol. 91, 2007. 